\title{
Cardinality enhancement utilizing Sequential Algorithm (SeQ) code in OCDMA system
}

\author{
C. A. S. Fazlina ${ }^{1, *}$, C. B. M. Rashidi ${ }^{1}$, A. K. Rahman ${ }^{2}$ and S. A. Aljunid ${ }^{1}$ \\ ${ }^{1}$ Centre of Excellence, ${ }^{1}$ Advanced Communication Engineering, Centre of Excellent School of Computer \& Communication Eng, \\ Universiti Malaysia Perlis, (UniMAP) Kangar, 01000, Kangar, Perlis \\ ${ }^{2}$ Department of Electrical and Electronic Eng, Universiti Malaysia Sarawak, (UNIMAS), Sarawak
}

\begin{abstract}
Optical Code Division Multiple Access (OCDMA) has been important with increasing demand for high capacity and speed for communication in optical networks because of OCDMA technique high efficiency that can be achieved, hence fibre bandwidth is fully used. In this paper we will focus on Sequential Algorithm (SeQ) code with AND detection technique using Optisystem design tool. The result revealed SeQ code capable to eliminate Multiple Access Interference (MAI) and improve Bit Error Rate (BER), Phase Induced Intensity Noise (PIIN) and orthogonally between users in the system. From the results, SeQ shows good performance of BER and capable to accommodate 190 numbers of simultaneous users contrast with existing code. Thus, $\mathrm{SeQ}$ code have enhanced the system about $36 \%$ and $111 \%$ of FCC and DCS code. In addition, SeQ have good BER performance $10^{-25}$ at $155 \mathrm{Mbps}$ in comparison with 622 Mbps, 1 Gbps and 2 Gbps bit rate. From the plot graph, $155 \mathrm{Mbps}$ bit rate is suitable enough speed for FTTH and LAN networks. Resolution can be made based on the superior performance of SeQ code. Thus, these codes will give an opportunity in OCDMA system for better quality of service in an optical access network for future generation's usage
\end{abstract}

\section{Introduction}

There have three types of multiple accesses; there are TDMA (Time Division Multiple Access), FDMA (Frequency Division Multiple Access) and CDMA (Code Division Multiple Access) [1]. In communication, the demand for traffic information is leading to increase of capacity and functionality. The characteristics such as bandwidth, transmitted information and data bit rate in security, are critical to the evolution of the OCDMA system [2]. Access and multiplexing techniques have allowed the simultaneous existence of multiple users on the network sharing with the same optical domain. Optical Code Division Multiple Access (OCDMA) techniques can provide moderate security communication. OCDMA also allowed to have multiple number of users to access optical network by sharing the bandwidth mechanism [3]. The main advantages of OCDMA compared with others techniques are the high capacity for higher connectivity, have more flexible bandwidth usage, higher granularity and scalability within optical networks, capable improved crosstalk performance, asynchronous access, and have potential for improved system security as number of users

Corresponding author: eng_fazz@yahoo.com on the network has specific address code [4]. In OCDMA system, a bit of " 1 " is to be transmitted as an assigned code and a bit of " 0 " does not transmit anything [5]. In OCDMA, the most important consideration is code design. The code which is designed improperly and higher number of simultaneous users can be degraded the system performance due to presence Multi Access Interference (MAI). MAI is the interference from other users transmitting at the same time, which will limit the effective error probability with the presence of noise in the overall system [6]. In the overall system, MAI is the interference between user's transmissions at the same time, which will limit the effective error probability with the presence of noise [7]. In OCDMA, Phase Induced Intensity Noise (PIIN) is deeply related the MAI. PIIN will increase between code sequences because of inappropriate cross correlation among the address sequences. PIIN depends on the number of interfering users and cannot be improved by increasing the transmitted power [8]. Most codes have been proposed for OCDMA system such as Modified Double Weight (MDW), Modified Frequency Hopping (MFH), Flexible Cross Correlation (FCC), Dynamic Cyclic Shift (DCS) and Hadamard code, respectively. In this paper, a new algorithm called Sequential Algorithm (SeQ) code has been developed. This proposed code was developed based on Tridiagonal matrix code with parity of even and odd were involved in order to enhance the limitations occurs in the existing codes. 


\subsection{Development of Sequential Algorithm (SeQ) Code}

The new class of SeQ code design is concerned about mathematical preliminaries to design codes for any given number of users and weights to have minimum code length. Since, optical codes may be represented as vectors; linear algebra has been used for the design of these codes. The codes are treated as rows of a matrix of finite dimension; hence, the methods of design and analysis of matrix algebra is best suited in these cases. Since, the codes are unique, the code matrix is required to have a finite value of its rank for a given set of users. The in phase auto-correlation functions and the crosscorrelation functions of the codes can easily be defined in term by the dot and cross product of the rows of the matrix. The auto-correlation of each codeword $A_{i}=\left(a_{i}\right.$, $\left.\mathrm{a}_{2}, \mathrm{a}_{3}, . . \mathrm{a}_{\mathrm{N}}\right)$ and the cross-correlation between any two distinct codeword $A_{i}=\left(a_{1}, a_{2}, a_{3} . . a_{N}\right)$ and $B_{j}=\left(b_{1}, b_{2}, b_{3}\right.$ .. $\mathrm{b}_{\mathrm{N}}$ ), respectively. Optical code are family of $K$ (for $K$ users) binary $[0,1]$ sequences of length $N$, code weight $W$ (the number of " 1 " in each codeword) and the maximum cross-correlation, $\lambda_{x y m}$.

$$
\begin{aligned}
& \lambda_{a}(\tau)=\sum_{i=1}^{N} a_{i} a_{i+\tau}=W \text { for } \tau=0 \\
& \lambda_{a b}(\tau)=\sum_{i=1}^{N} a_{i} b_{i+\tau} \leq 1 \text { for } \tau=0
\end{aligned}
$$

Hence, major bottleneck in the successful implementation of all optical networks is basically when all the users try to transmit the data simultaneously. It is noteworthy when designing coding sequences such that, it must cause least overlapping between data chips. Now, let $A=\left\{a_{\mathrm{n}}\right\}$ and $B=\left\{b_{\mathrm{n}}\right\}$ be the sequences of length $N$ such that the vector form can be written as [8];

$$
\left.\begin{array}{l}
\left\{a_{n}\right\}={ }^{\prime} 0^{\prime} \text { or }{ }^{\prime} 1^{\prime}, i=0, \ldots N-1 \\
\left\{b_{n}\right\}={ }^{\prime} 0^{\prime} \text { or } 1^{\prime}, i=0, \ldots N-1
\end{array}\right\}
$$

Since $x_{\mathrm{n}}$ is a $\{0,1\}$ binary sequence, the maximum value of $\lambda_{a}(\tau)$ in Eq (1) is for $\tau=0$ and is equal to $W$, the code weight of the sequence can be expressed as;

$$
\lambda_{a}(0)=W
$$

The codes described by Eq (3) can also be represented in vector form as;

$$
\left.\begin{array}{l}
A=\left\{a_{i}\right\}=\text { for } i=0,1, \ldots N-1 \\
B=\left\{b_{i}\right\}=\text { for } i=0,1 \ldots N-1
\end{array}\right\}
$$

where, $X$ and $Y$ are vectors of length $N$ with elements as defined by $\mathrm{Eq}$ (5). In terms of the vectors $A$ and $B$, Eqs (1) and (2) can be written as;

$$
\begin{aligned}
& \lambda_{a}(0)=a a^{T}=W \\
& \lambda_{a b}(0)=a b^{T}
\end{aligned}
$$

where, $A^{\mathrm{T}}$ and $B^{\mathrm{T}}$ denote the transpose of vectors $A$ and $B$, respectively.

The code weight of the codes is given by the inner (dot) product of the vector representing the codes. The length of the codes can also be controlled by controlling the number of columns of the code matrix with the present of bit parity of even and odd elements. The first $A_{i}^{\text {th }}$ row for the first $K$ user of the code matrix can be written as;

$$
A_{i}=\overbrace{11 . .1}^{W} \overbrace{0 . .0}^{x_{i}}
$$

where, the first row of $\mathrm{A}_{i}$ is define as;

$$
W+x_{i} \quad \text { for } 1,2, \ldots K
$$

Assume that, $r=\left(W-\lambda_{a b}\right)$ and $x_{i}$ can be derived as

$$
\begin{gathered}
x_{i}=K r+\lambda_{a b}-r(i-1)-W \\
=r(K-i)
\end{gathered}
$$

In summary, all the parameters of the codes can be defined in term of the matrix representing the codes set. When a set of code parameters is chosen, a code can be constructed with good auto and cross-correlation so that the interference called MAI and PIIN among users can be suppressed effectively by decoding the signals. This requires that the address codes must satisfy, where all address code word maybe easily distinguished from a possibly shifted version of every other code word.

From the basis of the above discussions, the construction of an optical codes having value of $\mathrm{K}, w, \lambda_{\mathrm{a}}$ consists of the following steps:

1). For a given number of users $\mathrm{K}$, and weight $w$, form a set of Non-Zero In Phase Cross Correlation code with minimum length as given by Eq (7).

2). The $\mathrm{K}$ rows of the code matrix that give the $\mathrm{K}$ optical CDMA codes having Non- Zero InPhase Cross-Correlation (IPCC), weight $w$ and minimum length.

This procedure will now be explained with the help of an example:

Assume that, it is desired to generate a set of minimum length of SeQ code for users $=4$, weight $=3$ and $\lambda_{\mathrm{a}}$ equal $=1$.

Table 1. Codeword of $\mathrm{SeQ}$ for $W=3, K=4$ and $\lambda_{2} \leq 1$

\begin{tabular}{|c|c|}
\hline Basic & Parity \\
\hline$(124)$ & 1101000 \\
$(235)$ & 0110100 \\
$(346)$ & 0011010 \\
$(457)$ & 0001101 \\
\hline
\end{tabular}

Notice that the code weight of each row more than two, and the relation between $\mathrm{N}$ and $\mathrm{K}$ for $\mathrm{SeQ}$ code can be express as:

$$
\mathrm{N}=\mathrm{K}+2 W-3
$$

\subsection{Signal to Noise Ration Performance Analysis}

The Signal Noise Ratio (SNR) is defined as the average signal to noise power, $\operatorname{SNR}=\left[\frac{I^{2}}{\sigma^{2}}\right]$, where $\sigma^{2}$ is the average power of noise which is given by,

$$
\sigma^{2}=\left\langle i_{\text {shot }}^{2}\right\rangle+\left\langle i_{\text {PIIN }}^{2}\right\rangle+\left\langle i_{\text {thermal }}^{2}\right\rangle
$$

Eq. (10) can be expressed as,

$$
\sigma^{2}=2 e B I+I^{2} B \tau_{C}+\frac{4 K_{b} T_{n} B}{R_{L}}
$$


Where, $e$ is the electron charge, $I$ is the average photocurrent, $I^{2}$ is the power spectral density for $I, B$ is the noise equivalent of electrical bandwidth, $K_{b}$ is the Boltzmann constant, $T_{n}$ is the absolute receiver noise temperature and $R_{L}$ is the receiver load resistor. Power Spectrum Density (PSD) will be calculated and the photodiode current I and can be written as follows;

$$
I=\mathcal{R} \int_{0}^{\infty} G(v) d v
$$

$\mathcal{R}$ represents as the responsively of the photo-detectors. Hence the photo current $I$ can be expressed as;

$$
I=\Re\left[\frac{P_{s r}[W]}{N}\right]
$$

The power of Shot noise can be written as;

$$
\left\langle i_{\text {Shot }}^{2}\right\rangle=2 e B \mathcal{R}\left[\frac{P_{S r}}{N}\right][3 W+1]
$$

The Phase Induced Intensity Noise (PIIN) noise will dominate the broadband sources. Hence, with PSD from each user is the same; the calculation for receiver PIIN noise directly from the total PSD of each photodiode. By using an Eq. (15) the PIIN noise at the receiver output is given by;

$$
\left\langle i_{P I I N}^{2}\right\rangle=B\left(I_{1}^{2} \tau_{c 1}+I_{1}^{2} \tau_{c 2}\right)=I^{2} * \tau_{C} * B
$$

Where, $I_{1}$ and $I_{2}$ are the average photodiode currents, $\tau_{c l}$ and $\tau_{c 2}$ are the coherence times of light incident on each photodiode. The variance of the PIIN noise at the receiver can be expressed as;

$$
\left\langle i_{P I I N}^{2}\right\rangle=\frac{B \mathcal{R}^{2} P_{S r}^{2} K W}{N^{2} \Delta_{V}}[3 W+1]
$$

Since all equation shown the properties of SeQ code are unique and independent of each other, Eq. (17) is also independent of the active users' data, consequently, proposed coding system does not depend on the timing of transitions in the data and it applied to the asynchronous system. Thermal noise is given as;

$$
\left\langle i_{\text {Thermal }}^{2}\right\rangle=\frac{4 K_{b} T_{n} B}{R_{L}}
$$

From Eqs. (14), (15), and (17), the SNR for the proposed SeQ code in the ODCMA coding system is defined by;

$$
S N R=\frac{\left[\frac{\Re P_{s r} W}{N}\right]^{2}}{\left[\frac{2 e B \Re P s r}{N}\right][3 W+1]+B \Re\left[\frac{P_{S r K W}^{2}}{N^{2} \Delta V}\right][3 W+1]+\frac{4 K_{b T_{n} B}}{R_{L}}}
$$

Since, there no pulses are sent for the data bit ' $O$ ' an assuming that the noise distribution is Gaussian, thus, the corresponding BER can be obtained as follows;

$$
B E R=\frac{1}{2} e f r c\left(\sqrt{\frac{S N R}{8}}\right)
$$

Finally, Eqs. (19) and (20) will be used for the numerical calculation for an evaluation of the proposed OCDMA coding system utilizing SeQ code. The performance of the $\mathrm{SeQ}$ code has been compared numerically with the existing OCDMA codes such as FCC and DCS codes. The numerical parameters used in calculations are shown in Table 2.

Table 2: Typical parameters for theoretical calculations.

\begin{tabular}{|c|c|}
\hline Parameter & Value \\
\hline Electron's charge & $e=1.60217646 \times 10^{-19}$ coulombs \\
\hline PD quantum & $\eta=0.75$ \\
\hline Electrical bandwidth & $\mathrm{B}=80 \mathrm{MHz}$ \\
\hline Boltzmann constant & $K_{b}=1.38 \times 10^{-23} \mathrm{~W} / \mathrm{K} / \mathrm{Hz}$ \\
\hline
\end{tabular}

\begin{tabular}{|c|c|}
\hline Receiver noise & $\mathrm{T}_{\mathrm{n}}=300 \mathrm{~K}$ \\
\hline Receiver load resistor & $\mathrm{R}_{\mathrm{L}}=1030 \Omega$ \\
\hline Data transmission rate & $\mathrm{R}_{\mathrm{b}}=155 \mathrm{Mbps}$ \\
\hline Broadband line width & $\Delta \lambda=3.75 \mathrm{THz}$ \\
\hline
\end{tabular}

\section{Results and Discussion}

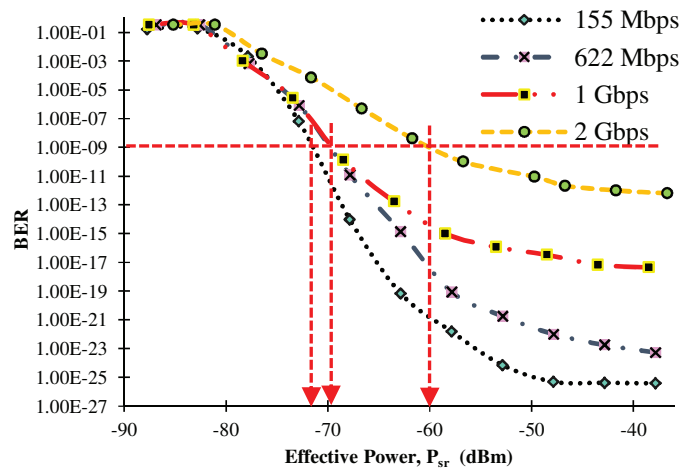

Fig. 1. Performance BER versus Effective Power, $\mathrm{P}_{\mathrm{sr}}(\mathrm{dBm})$ of $155 \mathrm{Mbps}, 622 \mathrm{Mbps}, 1 \mathrm{Gbps}$ and $2 \mathrm{Gbps}$ for SeQ (W=4) code.

Fig. 1 illustrates the plots of performance of BER reflect to the effective power, $\mathrm{P}_{\mathrm{sr}}(\mathrm{dBm})$ of varied 155 Mbps, 622 Mbps, 1 Gbps and 2Gbps for SeQ code. These result was analyzed by utilizing Optisystem, respectively. The result shows, the effective power $\mathrm{P}_{\mathrm{sr}}$ obtained from $-90 \mathrm{dBm}$ until $-30 \mathrm{dBm}$. From the results, the performance of BER for $155 \mathrm{Mbps}$ shows the excellent performance in comparison with $622 \mathrm{Mbps}, 1$ Gbps and 2Gbps. When the effective power, $\mathrm{P}_{\mathrm{sr}}$ larger $\left(\mathrm{P}_{\mathrm{sr}}>-72 \mathrm{dBm}\right)$ the performance of BER are increasingly decrease in comparison with when effective power $\mathrm{P}_{\mathrm{sr}}$ lower $\left(\mathrm{P}_{\mathrm{sr}}<-72 \mathrm{dBm}\right)$ the BER performance are worse. At communication error floor $\mathrm{BER}=10^{-09}$, the effective received power $\mathrm{P}_{\mathrm{sr}}$ are $-72 \mathrm{dBm},-60 \mathrm{dBm}$ for $155 \mathrm{Mbps}$ and $1 \mathrm{Gbps}$ data bit rate. Whereas, the effective received power $\mathrm{P}_{\mathrm{sr}}=-69 \mathrm{dBm}$ for data bit rate $622 \mathrm{Mbps}$ and $1 \mathrm{Gbps}$. From the plots graph, $155 \mathrm{Mbps}$ bit rate are suitable enough speed for FTTH and LAN networks.

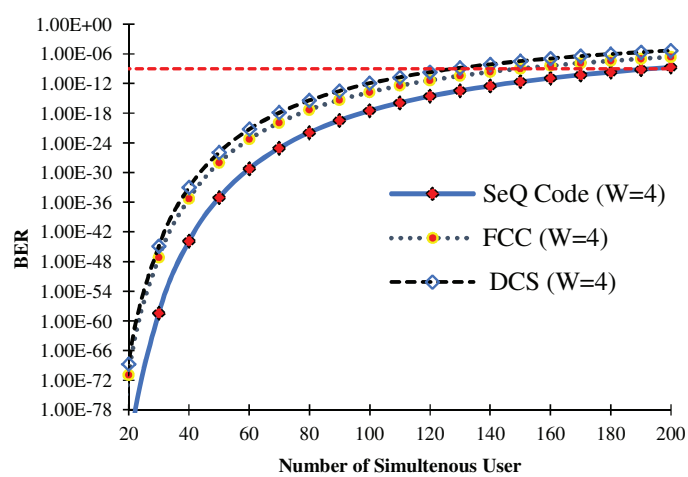

Fig. 2. Performance BER versus Number of Simultaneous Users, $\mathrm{K}$ for various OCDMA codes.

The plots graph demonstrates performance of BER versus number of simultaneous users, $\mathrm{K}$ for $\mathrm{SeQ}(\mathrm{W}=4)$, 
FCC $(\mathrm{W}=4)$ and DCS $(\mathrm{W}=4)$ OCDMA codes considered of 200 number of users at effective power, $\mathrm{P}_{\mathrm{sr}}-10 \mathrm{dBm}$ and $155 \mathrm{Mbps}$ bit rate, respectively. At typical error rate of optical communication system BER $10^{-09}$, the system performance of BER become increasingly degrade as the number of simultaneous users increased. This performance analyzed from Eq. (20). Fig. 2 shows performance of $\mathrm{SeQ}$ was superior in comparison with FCC $(\mathrm{W}=4)$ and DCS $(\mathrm{W}=4)$. Whereas, SeQ Code $(\mathrm{W}=4)$ can accommodate 190 number of users compared contrast to FCC $(\mathrm{W}=4)$ and DCS $(\mathrm{W}=4)$ can support 140 and 90 number of users, respectively. Since each code sequence is assigned to specific BER $=10^{-09}$ error floor, the SeQ $(\mathrm{W}=4)$ code has cardinality enhancement about $36 \%$ and $111 \%$ of FCC $(\mathrm{W}=4)$ and DCS $(\mathrm{W}=4)$. Thus, the SeQ code presents excellent performance due to the arrangement of the code algorithm and phase crosscorrelation. This performance of this system is revealed the improvement significantly, because the total loss is reduced as the AND detection technique requires less number of filters in the decoder. Hence, this results as an evidence that $\mathrm{SeQ}$ code can enhance the performance of the OCDMA system better than others existing codes.

\section{Conclusions}

The performance of OCDMA system by utilizing SeQ code have been performe. The system degradation due to PIIN can be suppressed by using SeQ code results in enhancing BER performance. The proposed SeQ code have excellent of BER performance contrast with effective received power, $\mathrm{P}_{\mathrm{sr}}$ based on the data bit rate where $155 \mathrm{Mbps}, 622 \mathrm{Mbps}, 1 \mathrm{Gbps}$ and 2 Gbps. The performance of the proposed $\mathrm{SeQ}$ code achieves high cardinality number of simultaneous user's in comparison with FCC (W=4) and DCS (W=4) code. Moreover, the $\mathrm{SeQ}$ code arrangement with less cross correlation value able to mitigates the PIIN and MAI, which capable to enhance the overall system performance.

This project was supported by the Government of Malaysia under the Fundamental Research Grant Scheme at Universiti Malaysia Perlis, Malaysia under the research grant \# 900300456.

\section{References}

1. L. Verma and G. Singh, Int. J. of Sci., Eng. And Tech. Res.3,6 (2014).

2. G. D. F. Guimarães, J. Electromagn. Anal. Appl., 5, 2, pp. 49-57, (2013).

3. T. H. Abd, S. A. Aljunid, H. A. Fadhil, R. A. Ahmad, and N. M. Saad, Opt. Fiber Technol., 17, 4, pp. 273-280, (2011).

4. A. O. Aldhaibani, S. A. Aljunid, M. S. Anuar, A. R. Arief, and C. B. M. Rashidi, Opt. Fiber Technol., 22, pp. 7-12, (2015).

5. S. A. Aljunid, F. Ghani, H. A. Fadhil, and M. S. Anuar, “ J. of Applied Sci. Res., 8,12, 5614-5626, (2012).

6. T. H. Abd, S. A. Aljunid, H. A. Fadhil, I. F. Radhi, and N. M. Saad, J. Opt. Comm., 33, 4, pp. 279-283, (2012).

7. C. B. M. Rashidi, S. A. Aljunid, F. Ghani, H. A. Fadhil, M. S. Anuar, and A. R. Arief, Optik, 125, 17, pp. 4889-4894, (2014).

8. N. D. Keraf and M. N. Nurol, Proceeding of Malaysia University Conference Engineering Technology 2014, pp 463-467,(2015). 